\title{
ALIMENTACIÓN DE LOS TRABAJADORES DEPENDIENTES RESIDENTES EN MONTEVIDEO Y CIUDADES CERCANAS
}

\section{FEEDING OF DEPENDENT WORKERS LIVING IN MONTEVIDEO AND NEARBY CITIES}

\author{
Claudia Suárez S., Andrea Echegoyen R., María Cerdeña Ch., \\ Verónica Perrone P., Isabel Petronio P.
}

Escuela de Nutrición y Dietética, Departamento de Administración de Servicios de Alimentación y Nutrición.

Departamento de Nutrición Social, Universidad de la República Oriental del Uruguay. Uruguay.

\begin{abstract}
The objective of the research was to learn the characteristics of Uruguayan workers'feeding, their health situations and the context and considerations that their companies offer to the consumption of food. The descriptive and cross-sectional type of the study allowed to collect data, through a telephone survey $(n=295)$, on relevant aspects of eating behavior. Almost all the workers had a meal time in hours of work and lunch was the meal that prevailed. Half of them chose red meat-based food followed by cooked vegetables as a second choice. Around $40 \%$ of workers said they had diet-related pathologies. Only $8 \%$ used the time recommended by the WHO for food consumption at work. Some companies do not respect the regulations of the Uruguayan government on the necessary conditions for the feeding of their employees. The implementation of policies that consider the feeding of the workers as a fundamental right is necessary.
\end{abstract}

Key words: feeding, health, rights.

Este trabajo fue recibido el 9 de Julio de 2010 y aceptado para ser publicado el 12 de Marzo de 2011.

\section{INTRODUCCIÓN}

La alimentación es un proceso vital, a través del cual las personas seleccionan, de la oferta de su entorno, los alimentos que conforman la dieta; en este sentido, la alimentación se considera un proceso voluntario y educable. Ha sido consagrada en la Declaración Universal de los Derechos Humanos del año 1948 (1) y en posteriores pactos, conferencias y cumbres, como un derecho humano fundamental.

En general, éste no ha sido considerado un tema de interés en el ámbito laboral; los estudios internacionales sobre la alimentación de los trabajadores son escasos y no existe ninguna investigación reciente en Uruguay relacionada con este aspecto. Sin embargo, las características y condiciones en que se alimentan los trabajadores son determinantes de su salud y del desarrollo de un trabajo digno, seguro y productivo. Actualmente la temática preocupa a las organizaciones y se reconoce como un aspecto sustancial para el diálogo entre el gobierno, los empleadores y los empleados (2).

Su relevancia es aún mayor al considerar las enfermedades no transmisibles relacionadas a la dieta, las que representan el $46 \%$ de la morbilidad y el $60 \%$ de la mortalidad a nivel mundial.

De acuerdo a datos de la "Primera Encuesta Nacional de Factores de Riesgo de Enfermedades Crónicas no trasmisibles" (3), difundidos por el Ministerio de Salud Pública, la mayoría de los adultos uruguayos presentan, al menos, un factor de riesgo para desarrollar éstas patologías. Entre los factores de riesgo conductuales se destacan: el tabaquismo, el consumo perjudicial de alcohol, el sedentarismo y la dieta no saludable. Además, éste estudio señala que el $60 \%$ de la población adulta (entre 25 y 64 años), presenta sobrepeso u obesidad.

La Organización Mundial de la Salud advierte que estos problemas sanitarios en los trabajadores pueden 
provocar pérdidas económicas de hasta el $20 \%$ de la producción (4).

Ante la situación planteada, se ha asignado a la "alimentación de los trabajadores", un rol sustantivo en el desarrollo social y económico, y los organismos internacionales recomiendan a los países implementar acciones de promoción, dirigidas a los trabajadores, para estimular el consumo de una alimentación balanceada, y así prevenir efectivamente los factores que pueden atentar en contra de la salud y el desempeño de este grupo de población. Si se considera que el trabajador permanece un tercio del día o la mitad de sus horas de vigilia en su lugar de trabajo, éste es el ámbito propicio para desarrollar intervenciones vinculadas con los cuidados de la salud y especialmente aquellas relacionadas con la correcta alimentación (2).

Dado que Uruguay carecía de información sobre la situación alimentaria de su fuerza laboral, el grupo investigador tuvo la inquietud de generar conocimiento en el tema y se propuso desarrollar una investigación que abordara aspectos genéricos de la alimentación de este grupo de población.

Es de esperar, que a partir de los resultados expuestos, se desarrollen nuevas líneas de investigación que permitan profundizar y generar nuevos conocimientos y que éstos sean considerados insumos a la hora de establecer políticas alimentarias dirigidas a los trabajadores.

\section{OBJETIVOS \\ Objetivo general}

Conocer las características de la alimentación de los trabajadores dependientes residentes en el Area Metropolitana, en el período julio - setiembre del año 2009.

\section{Objetivos específicos}

1. Investigar sobre los tiempos de comida que realizan habitualmente los encuestados en los días de trabajo.

2. Analizar el consumo de alimentos y preparaciones de los trabajadores.

3. Indagar sobre las patologías crónicas vinculadas a la alimentación que presentan los trabajadores.

4. Identificar los motivos por los cuales seleccionan los alimentos y/o preparaciones que consumen en el trabajo.

5. Indagar acerca del lugar y el tiempo empleado para el consumo de alimentos en el horario de trabajo

6. Analizar las condiciones que ofrecen las empresas para el consumo de comidas.

\section{MATERIAL Y MÉTODOS}

Tipo de estudio: Cuantitativo descriptivo de corte transversal.
Universo: Los trabajadores dependientes entre 18 y 65 años, de ambos sexos, que trabajaban 8 horas al día o más y que residían en el área Metropolitana (Montevideo y localidades cercanas de los Departamentos de San Jośe y Canelones - región donde habita el 59\% de la población nacional/Instituto Nacional de Estadística/ Uruguay).

Muestra: Aleatoria de carácter probabilística de tipo sistemático, siendo el tamaño muestral de 300 personas. La selección de la muestra se realizó sobre el listado de la guía telefónica. La unidad de muestreo fue el hogar con teléfono fijo. De acuerdo a este criterio de selección, en primera etapa se eligieron páginas de la guía y de cada página se escogieron 2 teléfonos con un intervalo de 10. Se entrevistó a uno de los residentes del hogar que cumpliera con las características establecidas para la población objetivo.

Método de recolección de datos: La información necesaria para este estudio se obtuvo de la propia declaración de los trabajadores a partir de una encuesta realizada en forma telefónica, empleando un formulario elaborado y pre codificado por el equipo investigador.

Para recabar los datos alimentarios se aplicó el método de frecuencia de consumo de alimentos.

La encuesta incluyó preguntas sobre: tiempos de comida realizados por los trabajadores, consumo habitual de alimentos y/o preparaciones, frecuencia semanal de consumo de alimentos, presencia de patologías crónicas relacionadas con la alimentación, motivos que llevan a la selección de los alimentos que se consumen en el trabajo, tiempo y y lugar destinado para la ingesta de comidas en la jornada laboral, condiciones que ofrecen los empleadores para el consumo de comidas.

Para evaluar la calidad del instrumento, se aplicó una prueba piloto sobre 23 casos, seleccionados al azar.

Se obtuvo un total de 295 encuestas efectivas.

Análisis estadístico: Para el procesamiento de datos se empleó el programa estadístico SPSS.

Se aplicó la prueba estadística de chi cuadrado para determinar la posible asociación entre las variables: consumo de alimentos/sexo, motivos de consumo de alimentos/sexo, presencia de enfermedades/sexo, presencia de enfermedades/edad.

\section{RESULTADOS Y DISCUSIÓN Características de la alimentación de los trabajadores}

Al estudiar las comidas diarias que realizaban los trabajadores, se detectó que $43 \%$ omitía al menos una comida: $8 \%$ de los trabajadores no desayunaba, 5\% omitía el almuerzo o la cena y el $30 \%$ no merendaba.

En la actualidad, el horario de trabajo extenso y/o 
el multiempleo llevan a que las personas permanezcan gran parte del tiempo fuera del hogar y deban realizar algunas comidas en el lugar de trabajo. En este sentido, se identificó que el $98 \%$ de los encuestados realizaba una o más comidas durante el horario de trabajo, (tabla 1). Los tiempos de comida realizados con mayor frecuencia en el horario laboral fueron el almuerzo y la merienda.

Al analizar los alimentos incluídos por los trabaja-

\begin{tabular}{lcc} 
& TABLA 1 \\
& \multicolumn{2}{c}{ Tiempos de comida realizados en el trabajo } \\
\hline TIEMPOS DE COMIDA & FA & FR (\%) \\
\hline Ninguna & 6 & $2 \%$ \\
Desayuno, almuerzo, merienda, cena & 4 & $1,4 \%$ \\
Desayuno, almuerzo, merienda & 11 & $3,7 \%$ \\
Desayuno, almuerzo & 31 & $10,5 \%$ \\
Almuerzo, merienda, cena & 3 & $1 \%$ \\
Almuerzo, merienda & 58 & $19,7 \%$ \\
Almuerzo, cena & 7 & $2,4 \%$ \\
Almuerzo & 165 & $55,9 \%$ \\
Merienda & 10 & $3,4 \%$ \\
Total & 295 & $100 \%$ \\
\hline FA: Frecuencia absoluta. & FR (\%): Frecuencia relativa porcentual &
\end{tabular}

\section{TABLA 2}

Grupo de alimentos consumidos por los trabajadores en el desayuno y la merienda

GRUPOS DE ALIMENTOS

\begin{tabular}{llcc} 
& & Desayuno & Merienda \\
No contesta & FA & 0 & 3 \\
Infusiones & FR\% & 0 & $1,4 \%$ \\
& FA & 112 & 89 \\
Lácteos & FR\% & $41,2 \%$ & $42,6 \%$ \\
& FA & 196 & 116 \\
Frutas & FR\% & $72,1 \%$ & $55.5 \%$ \\
& FA & 26 & 20 \\
Dulces & FR\% & $9,6 \%$ & $9,6 \%$ \\
& FA & 17 & 26 \\
Manteca, margarina & FR\% & $6,3 \%$ & $12,4 \%$ \\
& FA & 26 & 44 \\
Cereales & FR\% & $9,6 \%$ & $21,1 \%$ \\
& FA & 188 & 125 \\
Fiambres & FR\% & $69,1 \%$ & $59 \%$ \\
& FA & 10 & 19 \\
\hline FA: Frecuencia absoluta. & FR (\%): Frecuencia relativa porcentual & $3,7 \%$ & $9,1 \%$
\end{tabular}


dores en el desayuno y la merienda, se identificó, que en ambos tiempos de comida, los preferidos fueron los lácteos, los cereales y las infusiones (tabla 2). En el desayuno, el $17 \%$ de los trabajadores incluyó sólo un alimento, siendo las infusiones el grupo más destacado. En el grupo de las infusiones, el mate representó la mitad de las menciones. El "mate amargo" (yerba mate sin agregado de azúcares) integra el patrón cultural de nuestro país (5).

Cuando el desayuno se compone únicamente de una infusión sin el agregado de azúcar el aporte energético es nulo y de esta manera, se continúa el ayuno nocturno hasta tanto se ingiera algún alimento que aporte nutrientes y energía.

\section{TABLA 3}

Tipos de alimentos y preparaciones que consumen los trabajadores en el almuerzo según sexo.

\section{TIPO DE ALIMENTOS Y \\ PREPARACIONES DEL ALMUERZO}

Carnes rojas

Verduras cocidas

Cereales

Tortas, tartas, pizzas, empanadas

Comidas de olla

Ensaladas

Comidas rápidas

Pollo

Frutas

Sopas

Pescado

Refuerzos, sandwiches

Tortillas, croquetas y soufflés

Lácteos

Repostería, alfajores

\section{FA \\ FR\%}

FA

FR\%

FA

FR\%

FA

FR\%

FA

FR\%

FA

FR\%

FA

FR\%

FA

FR\%

FA

FR\%

FA

FR\%

FA

FR\%

FA

FR\%

FA

FR\%

FA

FR\%

FA

FR\%

FA

FR\%

\section{Sexo del encuestado} Femenino

64

41,3

51

32,9

29

18,7

36

23,2

20

12,9

21

13,5

12

7,7

15

9,7

10

6,5

10

6,5

6

3,9

4

2,6

1

0,6

0

0

0

0

1

0,6
Masculino

67

54,0

30

24,2

44

35,5

19

15,3

25

20,2

13

10,5

12

9,7

\section{5}

4

8

6,5

2

1,6

5

4

1

0,8

1

0,8

1

0,8

1

0,8

0

0

\section{Total}

131

47,0

81

29

73,0

26,2

55

19,7

45

16,1

34

12,2

24

8,6

20

7,2

18

6,5

12

4,3

11

3,9

5

1,8

2

0,7

1

0,4

1

0,4

1

0,4

Test Chi - cuadrado: $\mathrm{p}<0,01$ FA: Frecuencia absoluta. $\quad$ FR $(\%)$ : Frecuencia relativa porcentua. 
Al estudiar el comportamiento alimentario de los trabajadores en el almuerzo, se identificó que casi la mitad $(47 \%)$ seleccionaba comidas en base a carne vacuna.Las carnes de pollo y pescado, concentraron únicamente el $11 \%$ de las menciones. El segundo grupo de alimentos mencionado fue el de las verduras, siendo las cocidas las de mayor preferencia (29\%). Los cereales (arroz y pastas) se ubicaron en tercer lugar con el 26\% de las menciones. El 20\% de los trabajadores optaba por preparaciones como tortas, tartas saladas, pizzas y empanadas. En menor proporción (16\%) se consumían preparaciones de olla (guisos, ensopados) y comidas rápidas, entre otras.

En éste tiempo de comida, los hombres preferían preparaciones con carnes rojas más que las mujeres (54\% vs. $41 \%$ ). A su vez, las mujeres se inclinaban más que los hombres por las tartas, tortas saladas, pizzas y empanadas ( $23 \%$ vs. $15 \%$ ). (Chi - cuadrado: $\mathrm{p}<0,01$ (tabla 3).

El 4\% de los trabajadores cenaban en el horario laboral, destacándose un aumento en el consumo de sopas con respecto al almuerzo, lo cual podría vincularse con la estación del año en que se efectuó el estudio (invierno).

Al indagar en la frecuencia de consumo de alimentos, únicamente uno de cada tres trabajadores declaró consumir verduras a diario; en el caso de la frutas, el porcentaje de consumo diario asciende al $53 \%$.

En cuanto al consumo de carne roja (vacuna), el 26\%

\begin{tabular}{|c|c|c|c|c|c|c|}
\hline \multicolumn{6}{|c|}{ Frecuencia de consumo por grupo de alimentos. } & \\
\hline Frecuencia de consumo & & & & upo de Alimen & & \\
\hline & & Verduras & Frutas & Carnes rojas & Pollo & Pescado \\
\hline Nunca/casi nunca/esporádico & $\begin{array}{l}\text { FA } \\
\text { FR\% }\end{array}$ & $\begin{array}{c}5 \\
1,7\end{array}$ & $\begin{array}{l}29 \\
9,8\end{array}$ & $\begin{array}{c}30 \\
10,2\end{array}$ & $\begin{array}{c}44 \\
14,9\end{array}$ & $\begin{array}{c}144 \\
48,8\end{array}$ \\
\hline 1 ó 2 veces por semana & $\begin{array}{l}\text { FA } \\
\text { FR\% }\end{array}$ & $\begin{array}{l}62 \\
21\end{array}$ & $\begin{array}{c}46 \\
15,6\end{array}$ & $\begin{array}{c}82 \\
27,8\end{array}$ & $\begin{array}{c}175 \\
59,3\end{array}$ & $\begin{array}{l}127 \\
43,1\end{array}$ \\
\hline 3 ó 4 veces por semana & $\begin{array}{l}\text { FA } \\
\text { FR } \%\end{array}$ & $\begin{array}{l}130 \\
44,1\end{array}$ & $\begin{array}{c}63 \\
21,4\end{array}$ & $\begin{array}{c}106 \\
35,9\end{array}$ & $\begin{array}{c}61 \\
20,7\end{array}$ & $\begin{array}{c}23 \\
7,8\end{array}$ \\
\hline A diario & $\begin{array}{l}\text { FA } \\
\text { FR\% }\end{array}$ & $\begin{array}{c}98 \\
33,2\end{array}$ & $\begin{array}{c}157 \\
53,2\end{array}$ & $\begin{array}{c}77 \\
26,1\end{array}$ & $\begin{array}{l}15 \\
5,1\end{array}$ & $\begin{array}{c}1 \\
0,3\end{array}$ \\
\hline Totales & $\begin{array}{l}\text { FA } \\
\text { FR\% }\end{array}$ & $\begin{array}{l}295 \\
100\end{array}$ & $\begin{array}{l}295 \\
100\end{array}$ & $\begin{array}{l}295 \\
100\end{array}$ & $\begin{array}{l}295 \\
100\end{array}$ & $\begin{array}{l}295 \\
100\end{array}$ \\
\hline
\end{tabular}

TABLA 5

Presencia de enfermedades en los trabajadores según sexo

\begin{tabular}{llccc}
\hline \multicolumn{2}{l}{ PRESENCIA DE ENFERMEDADES } & \multicolumn{2}{c}{$\begin{array}{c}\text { Sexo del encuestado } \\
\text { Masculino }\end{array}$} & Total \\
& & Femenino & 55 & 114 \\
$\mathrm{Si}$ & FA & 59 & $42,3 \%$ & $38,6 \%$ \\
\multirow{2}{*}{$\mathrm{No}$} & FR\% & $35,8 \%$ & 75 & 181 \\
& FA & 106 & $57,7 \%$ & $61,4 \%$ \\
Total & FR\% & $64,2 \%$ & 130 & 295 \\
& FA & 165 & $100,0 \%$ & $100,0 \%$
\end{tabular}


de los encuestados manifestó consumirla diariamente; 1/3 parte la incluyó en su alimentación 3 ó 4 veces por semana. Para las carnes blancas (pollo y pescado), la frecuencia de consumo presentó diferencias: aproximadamente la mitad de la población encuestada consume pescado esporádicamente y el $60 \%$ incluye pollo en su alimentación una o dos veces a la semana (tabla 4).

Al comparar los resultados de este estudio con los de la Encuesta Nacional de Gastos e Ingresos de los Hogares de Uruguay 2005 - 2006 (6), se identificó que los trabajadores entrevistados presentan igual comportamiento que la población uruguaya al momento de seleccionar los tipos de carnes. La carne de vacuno es un alimento de consumo tradicional para los uruguayos, siendo la producción de ésta una de las principales actividades económicas del país.

Del análisis cualitativo de la alimentación de la población trabajadora, surge que en general, consumen

\begin{tabular}{|c|c|c|c|c|}
\hline & Presencia de enfermedades en los trabajadores según edad & $\begin{array}{l}\text { BLA } 6 \\
\text { en los trabaja }\end{array}$ & según edad & \\
\hline \multirow{2}{*}{\multicolumn{2}{|c|}{ PRESENCIA DE ENFERMEDADES }} & \multicolumn{2}{|c|}{ Tramos de edad } & \multirow[t]{2}{*}{ Total } \\
\hline & & 18 a 39 años & 40 a 65 años & \\
\hline $\mathrm{Si}$ & $\begin{array}{l}\text { FA } \\
\text { FR\% }\end{array}$ & $\begin{array}{c}28 \\
21,10 \%\end{array}$ & $\begin{array}{c}86 \\
53,10 \%\end{array}$ & $\begin{array}{c}114 \\
38,60 \%\end{array}$ \\
\hline No & $\begin{array}{l}\text { FA } \\
\text { FR\% }\end{array}$ & $\begin{array}{c}105 \\
78,90 \%\end{array}$ & $\begin{array}{c}76 \\
46,90 \%\end{array}$ & $\begin{array}{c}181 \\
61,40 \%\end{array}$ \\
\hline Total & $\begin{array}{l}\text { FA } \\
\text { FR\% }\end{array}$ & $\begin{array}{c}133 \\
100,0 \%\end{array}$ & $\begin{array}{c}162 \\
100,0 \%\end{array}$ & $\begin{array}{c}295 \\
100,0 \%\end{array}$ \\
\hline
\end{tabular}

\section{TABLA 7}

Tipo de enfermedades presentes en los trabajadores

TIPO DE ENFERMEDADES

Sobrepeso

Diabetes

Hipertensión arterial

Hipercolesterolemia

Enfermedades cardíacas

Osteoporosis

Hiperuricemia

Anemia

Total
FA

FR\%

FA

FR\%

FA

FR\%

FA

FR\%

FA

FR\%

FA

FR\%

FA

FR\%

FA

FR\%

FA

FR\%
Total

66

$22,4 \%$

16

$5,4 \%$

26

$8,8 \%$

42

$14,2 \%$

12

$4,1 \%$

2

$0,7 \%$

4

$1,4 \%$

4

$1,4 \%$

172

$100 \%$

FA: Frecuencia absoluta. FR (\%): Frecuencia relativa porcentual. 
a diario alimentos correspondientes a todos los grupos recomendados en las Guías Alimentarias Basadas en Alimentos (GABA) diseñadas para Uruguay (7).

\section{Presencia de enfermedades no trasmisibles vinculadas a la dieta}

Al indagar sobre las enfermedades relacionadas con la alimentación, el 39\% de los trabajadores expresó presentar alguna/s de ellas. De las mujeres, el 36\% manifestó padecer alguna de estas patologías; en el caso de los hombres, la proporción aumenta al 42\% (tabla 5).

La presencia de enfermedades mantiene relación con la edad de los trabajadores; a medida que aumenta la edad, mayor es la ocurrencia de enfermedades crónicas relacionadas con la alimentación (Test Chi - cuadrado: $\mathrm{p}<0,01$ ) (tabla 6).

Aproximadamente, el $25 \%$ de los entrevistados percibe que presenta sobrepeso, $14 \%$ expresa que padece hipercolesterolemia, 9\% hipertensión arterial, 5\% diabetes, $4 \%$ cardiopatías y $3 \%$ otras enfermedades como anemia, osteoporosis o hiperuricemia (tabla 7).

Los resultados obtenidos del análisis de esta variable para sobrepeso, presentan la misma tendencia que los hallados en la $1^{\circ}$ Encuesta Nacional de Factores de Riesgo de Enfermedades Crónicas no trasmisibles realizada en Uruguay (3). Éstas enfermedades pueden provocar, un deterioro en la salud física y mental, aumentando los costos de las acciones sanitarias y actuando en detrimento de la producción de los bienes y servicios que la sociedad requiere para subsistir y desarrollarse. Cuando la población trabajadora presenta esta patologías, además de verse afectada su calidad de vida y la de entorno, se identifican efectos adversos en la estructura socio-económica de los países, dado que los trabajadores constituyen la población económicamente activa (2).

\section{Condiciones de consumo de alimentos durante la jornada laboral}

Al indagar acerca de los motivos que llevan a la selección de los alimentos que se consumen en el horario

\section{TABLA 8}

Motivos de consumo de los alimentos que se ingieren en el trabajo según sexo.

\begin{tabular}{|c|c|c|c|c|}
\hline \multirow[t]{2}{*}{ MOTIVOS DE CONSUMO } & & \multicolumn{2}{|c|}{ Sexo del encuestado } & \multirow[t]{2}{*}{ Total } \\
\hline & & Femenino & Masculino & \\
\hline $\mathrm{NS} / \mathrm{NC}$ & $\begin{array}{l}\text { FA } \\
\text { FR\% }\end{array}$ & $\begin{array}{c}8 \\
4,8 \%\end{array}$ & $\begin{array}{c}3 \\
2,3 \%\end{array}$ & $\begin{array}{c}11 \\
3,7 \%\end{array}$ \\
\hline Practicidad, rapidez & $\begin{array}{l}\text { FA } \\
\text { FR\% }\end{array}$ & $\begin{array}{c}63 \\
38,1 \%\end{array}$ & $\begin{array}{c}63 \\
48,5 \%\end{array}$ & $\begin{array}{c}136 \\
42,7 \%\end{array}$ \\
\hline Cuidado por la salud. & $\begin{array}{l}\text { FA } \\
\text { FR\% }\end{array}$ & $\begin{array}{c}29 \\
17,6 \%\end{array}$ & $\begin{array}{c}15 \\
11,5 \%\end{array}$ & $\begin{array}{c}44 \\
14,9 \%\end{array}$ \\
\hline Es lo que ofrecen en el trabajo & $\begin{array}{l}\text { FA } \\
\text { FR\% }\end{array}$ & $\begin{array}{c}28 \\
17,0 \%\end{array}$ & $\begin{array}{c}11 \\
8,5 \%\end{array}$ & $\begin{array}{c}39 \\
13,2 \%\end{array}$ \\
\hline Lo lleva del hogar. & $\begin{array}{l}\text { FA } \\
\text { FR\% }\end{array}$ & $\begin{array}{c}14 \\
8,5 \%\end{array}$ & $\begin{array}{c}22 \\
16,9 \%\end{array}$ & $\begin{array}{c}36 \\
12,2 \%\end{array}$ \\
\hline Económico & $\begin{array}{l}\text { FA } \\
\text { FR\% }\end{array}$ & $\begin{array}{c}11 \\
6,7 \%\end{array}$ & $\begin{array}{c}4 \\
3,1 \%\end{array}$ & $\begin{array}{c}15 \\
5,1 \%\end{array}$ \\
\hline Preferencia & $\begin{array}{l}\text { FA } \\
\text { FR\% }\end{array}$ & $\begin{array}{c}3 \\
1,8 \%\end{array}$ & $\begin{array}{c}9 \\
6,9 \%\end{array}$ & $\begin{array}{c}12 \\
4,1 \%\end{array}$ \\
\hline Por costumbre & $\begin{array}{l}\text { FA } \\
\text { FR\% }\end{array}$ & $\begin{array}{c}5 \\
3,0 \%\end{array}$ & $\begin{array}{c}3 \\
2,3 \%\end{array}$ & $\begin{array}{c}8 \\
2,7 \%\end{array}$ \\
\hline En el descanso va a comer a la casa & $\begin{array}{l}\text { FA } \\
\text { FR\% }\end{array}$ & $\begin{array}{c}4 \\
2,4 \%\end{array}$ & $\begin{array}{c}0 \\
0,0 \%\end{array}$ & $\begin{array}{c}4 \\
1,4 \%\end{array}$ \\
\hline Total & $\begin{array}{l}\text { FA } \\
\text { FR\% }\end{array}$ & $\begin{array}{c}165 \\
100,0 \%\end{array}$ & $\begin{array}{c}130 \\
100,0 \%\end{array}$ & $\begin{array}{c}295 \\
100,0 \%\end{array}$ \\
\hline
\end{tabular}


laboral, el $43 \%$ de los encuestados mencionó lo "práctico". Este motivo pudiera relacionarse con el tiempo que disponen los trabajadores para realizar las comidas en el horario laboral o por tener un fácil acceso a los alimentos, en caso que la empresa los ofrezca o por la oferta en comercios cercanos.

Al estudiar este aspecto según el sexo del trabajador, se identificó que los hombres mencionan este motivo con más frecuencia que las mujeres (48\% vs. $38 \%)$. Test Chi - cuadrado: $\mathrm{p}<0,01$ (tabla 8).

Otros trabajadores expresaron: cuidado de la salud (15\%), oferta de alimentos por parte de la empresa (13\%) y suministro de alimentos provenientes del hogar (12\%).

En relación al tiempo que los trabajadores destinan para el consumo de comidas en el trabajo, más de la mitad expresó emplear menos de 40 minutos para comer. En este sentido, la OMS sugiere que se dedique no menos de 40 minutos para consumir una de las comidas principales en el trabajo (8). El 6\% utilizaba menos de 10 minutos, situación que puede provocar en los trabajadores trastornos generales y digestivos. Entre los trastornos generales se destacan: la hipertensión arterial, la falta de atención y la somnolencia. Éstos afectan el rendimiento laboral y aumentan la probabilidad de sufrir accidentes.

El lugar empleado para consumir los alimentos en el trabajo juega un rol muy importante en el mantenimiento de la salud. Dicho ambiente debe permitir a las personas renovar y crear vínculos con sus compañeros, liberar tensiones y desvincularse temporalmente del puesto de trabajo. Aunque el $78 \%$ de los encuestados expresó que la empresa donde trabajan dispone de comedor, únicamente el $57 \%$ lo utiliza a la hora de consumir las comidas. El 26\% de los trabajadores come en el mismo lugar donde desempeña las tareas; en este caso, el puesto de trabajo se convierte en "comedor", con los riesgos psíquicos y biológicos que esto implica, (gráfico 1).

Al estudiar la disponibilidad de equipos para refrigerar y calentar las comidas, se identificó que el $90 \%$ de los trabajadores contaba, en su trabajo, con refrigerador y el $93 \%$ con equipamiento para cocinar o calentar las preparaciones. Cuatro de cada cinco trabajadores manifestaron disponer de microondas.

En este sentido, la disponibilidad de equipo necesario para adecuar las comidas para el consumo puede estar incidiendo en el tipo de preparaciones que se seleccionan para comer.

Uruguay cuenta con disposiciones reglamentarias vinculadas a la protección de la salud y la vida de los trabajadores donde se establecen las condiciones que deben ofrecer los empleadores para la alimentación en los lugares de trabajo (9). Las mismas hacen referencia a que los establecimientos, en donde se cumplan jornadas de trabajo en el régimen de horario continuo, deben contar

\section{GRAFICO 1}

Lugar de consumo de alimentos y preparaciones durante el horario de trabajo

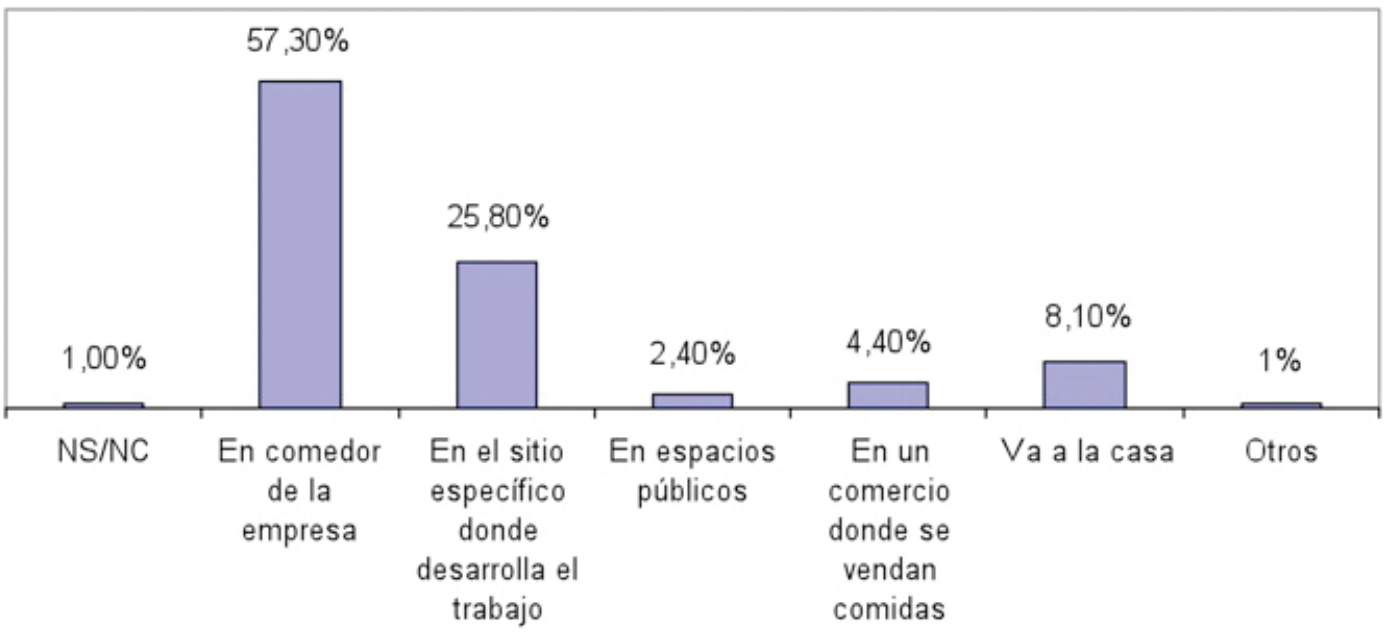


o facilitar un local destinado a comedor. Además, deben procurar a los trabajadores los medios para conservar en lugares apropiados los alimentos que han llevado consigo, así como equipos para poder calentarlos.

De acuerdo a los resultados de esta investigación, algunas de las empresas donde se desempeñan los trabadores encuestados no cumplen con los servicios dispuestos en la reglamentación vigente.

En relación a la oferta de servicio de comidas por parte de la empresa, sólo $29 \%$ de los trabajadores mencionó que en su trabajo dispone de éste servicio. La reducida oferta por parte de las empresas puede estar vinculada a las siguientes causas:

- Que los empleadores no hayan evaluado los beneficios que servicios de estas características pueden tener para los trabajadores (practicidad en lo que respecta al acceso a los alimentos, ahorro de tiempo y dinero, consumo de alimentos nutritivos e inocuos) e indirectamente, para la propia organización.

- Que los empleadores no hayan encontrado la manera de facilitar la comida a los empleados que mejor se adapte a las condiciones de la organización (tamaño, situación financiera, localización)

- Que los trabajadores, desde sus sindicatos, no reclamen por este beneficio, por no considerarlo un tema prioritario en las mejoras de las condiciones laborales.

- Que el Estado no ofrezca suficientes incentivos para que las empresas ofrezcan servicios de comidas saludables a sus empleados.

De acuerdo a los resultados obtenidos en esta investigación se puede concluir que:

- Dada la trascendencia que adquiere el tema para las personas, las organizaciones y el país, se considera primordial, promover una alimentación completa y armónica en los trabajadores, lo cual constituye una responsabilidad compartida entre el gobierno, las empresas y los sindicatos.

- El plan de acción para revertir los aspectos negativos de la situación alimentaria encontrada debe estar basado en el principio de que todos los trabajadores deben poder disfrutar del más alto nivel posible de salud y de condiciones de trabajo que actúen en pro de su bienestar. En este sentido, la educación alimentario nutricional constituye un pilar fundamental en todos los sectores implicados, promoviendo la alimentación saludable en el trabajo y mejorando la calidad de vida de las personas.

\section{RESUMEN}

El objetivo de la investigación fue conocer las características de la alimentación de los trabajadores/ as uruguayos, su situación de salud y las condiciones ofrecidas por empleadores para el consumo de comidas. Este estudio descriptivo de corte transversal, permitió recabar mediante una encuesta telefónica $(n=295)$, datos relevantes del comportamiento alimentario. Casi el total de trabajadores, realizaban alguna comida en horario laboral, predominando el almuerzo. La mitad de ellos seleccionaban preparaciones con carnes rojas, le seguían los vegetales cocidos. Aproximadamente, el $40 \%$ de los trabajadores declaró presentar patologías relacionadas con la dieta. Únicamente el $8 \%$ destinaba el tiempo recomendado por la OMS para el consumo de alimentos en el trabajo. Algunas empresas incumplen las normativas del Gobierno Uruguayo sobre las condiciones necesarias para la alimentación de sus empleados. Continuar investigando en la temática implica hacer camino en la instauración de políticas que contemplen la alimentación de los trabajadores como un derecho fundamental.

Palabras clave: alimentación, salud, derechos.

Dirigir la correspondencia a:

Profesora

Claudia Suárez S.

Escuela de Nutrición y Dietética

Departamento de Administración de Servicios

de Alimentación y Nutrición.

Universidad de la República Oriental del Uruguay

Yaguarón 1542, Apto. 901

Montevideo, Uruguay.

Teléfono. 099418315

E-mail: claususil@yahoo.com

\section{BIBLIOGRAFÍA}

1. Declaración Universal de Derechos Humanos. Artículo 25. 1948.

2. Christopher Wanjek. "La alimentación en el trabajo: soluciones laborales para la desnutrición, la obesidad y las enfermedades crónicas". Organización Internacional del Trabajo. Ginebra. 2005.

3. Ministerio de Salud Pública. Dirección General de la Salud. División Epidemiología. $1^{\text {a Encuesta }}$ Nacional de Factores de Riesgo de Enfermedades Crónicas No Transmisibles. Uruguay. 2008.

4. World Health Organization. Recommendation of the second meeting of WHO Collaborating Centres in Occupational Health. Beijing, China. 1995.

5. Salazar Ruben et al. El Mate: Símbolo de identidad nacional. Premio Nacional de Nutrición. Ministerio de Trabajo y Seguridad Social. Instituto Nacional de Alimentación. Montevideo (Uruguay). 1999.p. $43-7$.

6. Instituto Nacional de Estadística. Los alimentos 
y bebidas en los hogares. Encuesta Nacional de Gastos e Ingresos de los Hogares 2005- 2006. Uruguay. 2008

7. Grupo Interinstitucional de trabajo para las Guías Alimentarias basadas en alimentos de Uruguay. Manual para la Promoción de Prácticas Saludables de Alimentación en la Población Uruguaya. Min- isterio de Salud Pública. Programa Nacional de Nutrición. Uruguay, 2005

8. Serra Majem L et. al. Nutrición y Salud Pública. Métodos, bases científicas y aplicaciones. Editorial Masson S.A. España. 2006.

9. Decretos. $\mathrm{N}^{\circ} 406 / 88, \mathrm{~N}^{\circ} 89 / 1995, \mathrm{~N}^{\circ} 372 / 99$. Poder Ejecutivo. Republica Oriental del Uruguay. 